\title{
Composite absorbing potentials
}

\author{
J. P. Palao, J. G. Muga and R. Sala \\ Departamento de Física Fundamental y Experimental, Universidad de La Laguna, Tenerife, Spain
}

\begin{abstract}
The multiple scattering interferences due to the addition of several contiguous potential units are used to construct composite complex potentials that absorb at an arbitrary set of incident momenta or for a broad momentum interval.
\end{abstract}

PACS: 03.65.Nk, 34.90.+q

Complex absorbing potentials are an important tool in stationary or time dependent scattering calculations [四]. They avoid spurious edge effects of the finite "box" where the system is enclosed for numerical purposes in wave packet calculations [2]. They have been also used in other contexts, such as time-independent approaches to reactive scattering [3] 5], or calculations of the microcanonical cumulative reaction probability, and transition probabilities in time dependent fields [6,7]. Allcock, and other authors have claimed that no perfectly absorbing potential can exist, even for one single incident momentum, with finite spatial support [8]. But counterexamples have been found, i.e., potentials that absorb perfectly at one selected momentum for an arbitrarily small support do exist [9]. In actual collisions, however, the wave packets may have broad translational momentum distributions and be peaked around different momenta. Such a case arises, for example, as a result of internal state energy discretization of the collision products [10]. So the challenge is to model complex potentials that absorb perfectly at a discrete set of energies, or with sufficient efficiency for broad momentum intervals.

In this letter we shall make use of the interferences between paths associated with "multi- 
ple collisions" in composite barriers to construct complex potentials that absorb at a selected set of incident momenta or for a broad momentum range. Two different complementary methods are described and demonstrated. The first method works by successive addition of one potential unit for each absorbed momentum and leads to perfect absorption for the selected momenta. In the second method no perfect absorption is achieved but it is numerically much more robust than the former, and allows for efficient absorption in a broad momentum range.

The first construction method is now described. Assume two complex potential units $V_{1}$ and $V_{2}$ of contiguous, finite supports, $V_{1}$ being the first one from the left, and $V_{2}$ the second. Let $T_{i}^{r, l}$ and $R_{i}^{r, l}, i=1,2$, be the complex transmission and reflection amplitudes for left $(l)$ and right $(r)$ incidence for the $i$-th (isolated) potential unit, and $T^{r, l}$ or $R^{r, l}$ (without subscript) the corresponding amplitudes for the compound barrier, $V=V_{1}+V_{2}$. These last quantities may be obtained in terms of the former by the "multiple collision" technique [11], i.e., by considering the sum of the amplitudes for all the possible "paths" that lead eventually to transmission or reflection. In particular,

$$
\begin{aligned}
& T^{l}=T_{1}^{l}\left[\sum_{n=0}^{\infty}\left(R_{2}^{l} R_{1}^{r}\right)^{n}\right] T_{2}^{l}=T_{1}^{l} \frac{1}{1-R_{2}^{l} R_{1}^{r}} T_{2}^{l} \\
& R^{l}=R_{1}^{l}+T_{1}^{l} R_{2}^{l}\left[\sum_{n=0}^{\infty}\left(R_{2}^{l} R_{1}^{r}\right)^{n}\right] T_{1}^{r}=R_{1}^{l}+T_{1}^{l} R_{2}^{l} \frac{1}{1-R_{2}^{l} R_{1}^{r}} T_{1}^{r}
\end{aligned}
$$

Now assume that

$$
T_{1}^{l}\left(k_{1}\right)=R_{1}^{l}\left(k_{1}\right)=0
$$

where $k_{1}>0$ is a particular (dimensionless) wave number. $\left(k=d_{1} p / \hbar\right.$, where $p$ is the dimensional momentum and $d_{1}$ the dimensional width of the first barrier). Inserting (2) into (1) gives $T^{l}\left(k_{1}\right)=R^{l}\left(k_{1}\right)=0$. In other words, if $V_{1}$ is a perfect absorber at $k_{1}$, the total potential $V$ is a perfect absorber for $k_{1}$ too. The objective of adding $V_{2}$ is to absorb also at $k_{2} \neq k_{1}$. If $V_{2}$ is naively constructed in such a way that $R_{2}^{l}\left(k_{2}\right)=T_{2}^{l}\left(k_{2}\right)=0$, according to the equations (1) the composite barrier does not transmit the new momentum, $T^{l}\left(k_{2}\right)=0$, 
but in general $R^{l}\left(k_{2}\right) \neq 0$ because of reflection in the first barrier, $R_{1}^{l}\left(k_{2}\right) \neq 0$. Instead, if the second potential satisfies

$$
\begin{aligned}
& T_{2}^{l}\left(k_{2}\right)=0, \\
& R_{2}^{l}\left(k_{2}\right)=\frac{R_{1}^{l}\left(k_{2}\right)}{R_{1}^{l}\left(k_{2}\right) R_{1}^{r}\left(k_{2}\right)-T_{1}^{l}\left(k_{2}\right) T_{1}^{r}\left(k_{2}\right)},
\end{aligned}
$$

total absorption is achieved at $k_{2}, T^{l}\left(k_{2}\right)=R^{l}\left(k_{2}\right)=0$. Eq. (44) is obtained by assuming $R^{l}\left(k_{2}\right)=0$ and solving for $R_{2}^{l}$ in (11) in terms of quantities that depend only on $V_{1}$. Even though the two barriers alone have non vanishing reflection amplitudes for $k_{2}$, when they are put together the interference effects exactly cancel the global reflection.

We shall next describe a way to construct $V_{1}$ and $V_{2}$ with the desired partial reflection and transmission amplitudes. Dimensionless variables will be used throughout. The dimensionless position $x$ is obtained by dividing the dimensional position by a reference length ( $d_{1}$ in this case), so that the support of $V_{1}$ has length one when using the $x$ variable. The second potential unit, $V_{2}$, may have a different length, $L_{2}=d_{2} / d_{1}$. Dimensionless energies (kinetic and potential) are obtained by dividing the corresponding dimensional quantities by $\hbar^{2} /\left(2 m d_{1}^{2}\right), m$ being the mass of the particle.

Potentials $V_{1}$ with vanishing reflection and transmission coefficients for $k_{1}$ can be constructed by means of an inversion procedure similar to the one described in [9]. The important difference with respect to that work is that now transmission is allowed at other wave numbers, so that the wave function and its derivative are continuous at $x=1$. This is necessary to take advantage of interference effects. The boundary conditions to be satisfied by the stationary wave function corresponding to an incident plane wave with wave number $k_{1}$ at the two potential edges are

$$
\begin{aligned}
& \psi_{1}(0)=1, \quad \psi_{1}^{\prime}(0)=i k_{1}, \\
& \psi_{1}(1)=\psi_{1}^{\prime}(1)=0,
\end{aligned}
$$

where the prime means "derivative with respect to $x$ ". To satisfy these four conditions the wavefunction between 0 and 1 is written in terms of a functional form with four free 
parameters. By substituting this expression in the four equations (5), the four parameters are determined, and solving in the Schrödinger equation for $V_{1}(x)$ one finds

$$
V_{1}=k_{1}^{2}+\psi_{1}^{\prime \prime} / \psi_{1}
$$

The simplest choice for $\psi_{1}$ is a polynomial, $\psi_{1}=\sum_{j=0,3} a_{j} x^{j}$. From (5) the coefficients $a_{j}$ are readily obtained,

$$
a_{0}=1, \quad a_{1}=i k_{1}, \quad a_{2}=-3-2 i k_{1}, \quad a_{3}=2+i k_{1}
$$

To construct the potential $V_{2}$ between 1 and $1+L_{2}$, or additional units with support between two points $x=z$ and $x=z+L$, it is convenient to define the new variable $y=(x-z) / L$, so that the potential unit goes from $y=0$ to $y=1$. A new wavenumber is also defined as $\hat{k}=L k$ (remember that $L$ is dimensionless). The reflection amplitude $r^{l}(\hat{k})$ for the new variables $(\hat{k}, y)$ is related to the one for the original variables $(k, x)$ by $r^{l}(\hat{k})=R^{l}(k) e^{-2 i k z}$ [12]. It is much easier to manipulate the constraints (3) and (4) using the new set of variables. In particular, for obtaining $V_{2}$, it is first assumed that the wave function $\Psi_{2}(y)$ corresponding to a plane wave incident from the left with wavenumber $\hat{k}_{2}=L_{2} k_{2}$ obeys the four conditions

$$
\begin{aligned}
& \Psi_{2}(y=0)=1+r_{2}^{l} \\
& \Psi_{2}^{\prime}(y=0)=i \hat{k}_{2}\left(1-r_{2}^{l}\right) \\
& \Psi_{2}(y=1)=\Psi_{2}^{\prime}(y=1)=0,
\end{aligned}
$$

with $r_{2}^{l}\left(\hat{k}_{2}\right)=R_{2}^{l}\left(k_{2}\right) e^{-2 i k_{2}}$ and $R_{2}^{l}\left(k_{2}\right)$ given by (州. By assuming, as before, a polynomial form, $\Psi_{2}(y)=\sum_{j=0,3} b_{j} y^{j}$, and substituting in (8), the coefficients are found to be

$$
\begin{aligned}
& b_{0}=1+r_{2}^{l}, \quad b_{1}=i \hat{k}_{2}\left(1-r_{2}^{l}\right), \\
& b_{2}=-\left(3+2 i \hat{k}_{2}\right)-r_{2}^{l}\left(3-2 i \hat{k}_{2}\right), \quad b_{3}=\left(2+i \hat{k}_{2}\right)+r_{2}^{l}\left(2-i \hat{k}_{2}\right) .
\end{aligned}
$$

Solving in the Schrödinger equation for the corresponding potential and rewriting the result for the original variables, $\psi_{2}(x)=\Psi_{2}(y)$, one then finds for $V_{2}$ between $x=1$ and $x=1+L_{2}$,

$$
V_{2}(x)=k_{2}^{2}+\frac{\psi_{2}^{\prime \prime}}{\psi_{2}} L_{2}^{2}
$$


The potential $V_{1}+V_{2}$ so constructed is a perfect absorber at $k_{1}$ and $k_{2}$, and addition of a third unit $V_{3}$ will not change this property, as we have already discussed for the addition of $V_{2}$. By treating the $V_{1}+V_{2}$ potential as a new $\widetilde{V_{1}}$ unit, and the new barrier $V_{3}$ as $\widetilde{V}_{2}$, the inversion method can be repeated to build a potential $V_{3}$ that absorbs $k_{3}$. This procedure can be continued to construct "perfectly absorbing composite potentials" for an arbitrary number of momenta. (With little changes the method is also applicable when an infinite barrier is put at the right edge of the last barrier. The only difference is that the condition $\psi^{\prime}\left(x=\sum L_{j}\right)=0$ need not be imposed so that a quadratic polynomial, rather than a cubic one, is enough for the last barrier.) Note that the polynomials and the minimal set of conditions discussed here have the advantage of providing simple explicit expressions but other functional forms for the wave functions may be used, and further conditions may be imposed.

Figure 1 shows the survival probability $S(k) \equiv\left|R^{l}(k)\right|^{2}+\left|T^{l}(k)\right|^{2}$ versus $k$ for potentials constructed with two units in this fashion (thick solid and dotted lines). The effective absorption width around $k_{2}$ increases with decreasing $L_{2}$, an effect reminiscent of the the broadening of transmission resonances when the walls of a double barrier are narrowed. In Figure 2 three units are used to absorb at three different wavenumbers. The prize to pay for the requirement of perfect absorption is that, at least for the potential units we have studied, numerical instabilities complicate their practical implementation. Even though the potential is explicit, the absorption at an arbitrary momentum has to be computed numerically. The numerically calculated $S$ at and around $k_{2}$ becomes very sensitive to small errors in the discretization of the potentials if $T_{1}\left(k_{2}\right)$ is extremely small 13 (It appears as a denominator in the estimate of the error of $S$ caused by errors in $R_{1}^{l, r}$ or $T_{1}$ ). A way to avoid this problem in practice is to truncate the potentials so that $S\left(k_{1}\right)$ is not exactly zero (but sufficiently close for practical purposes, say $\left.S\left(k_{1}\right)=10^{-5}\right)$. This increases $T_{1}\left(k_{2}\right)$ and makes $S$ in the proximity of $k_{2}$ much more stable with respect to slight numerical errors due to the discrete representation of the potential. However, the difficulties increase when adding more $k$ points. A numerically robust alternative is described next. 
The second method makes also use of interferences between contiguous units, but in a less explicit way than the former approach. Now the functional form chosen for the potential is a series of $N$ equal length complex square barriers with complex energies $\left\{V_{j}\right\}, j=1,2, \ldots, N$. The real and imaginary values of $V_{j}$ are optimized with standard subroutines according to a flexible criterion: The sum of the survivals for a set of $s$ selected points is minimized [14],

$$
f\left(V_{1}, \ldots, V_{N} ; k_{1}, \ldots, k_{s}\right)=\sum_{\alpha=1}^{s} S\left(V_{1}, \ldots, V_{N} ; k_{\alpha}\right)
$$

Note that $s$ and $N$ are not necessarily equal. In a generic application the $s$ points are evenly spaced in a given interval in order to absorb arbitrary wave packets within the interval. The advantage of this functional form for the potential is that, for a given set of values $\left\{V_{j}\right\}$, the total transmission and reflection coefficients and their gradients with respect to variations of the barrier parameters can be obtained exactly by multiplication of $2 \times 2$ transfer matrices. These evaluations are very fast, so that many more parameters can be optimized, two for each barrier, than for other functional forms [15,16].

In Figures 1 and 2 the survival probabilities obtained with the two methods for $s=2$ and $s=3$ can be compared in the low (dimensionless) wavenumber region, which is the important one to minimize the (dimensional) absorbing potential width in the applications. The second method provides with just a few barriers excellent survival curves below 0.001, which is sufficient for most practical purposes. Note the improvement of the survival curves as the number of barriers increases. This method achieves larger absorption widths than a previous systematic approach [9, 15] in all studied cases [16], with the added advantage of a very simple implementation. We have also included in both figures the survival curves for one of the most frequently used potentials, $V=-i \eta x^{2}$, where $\eta$ (real) has been chosen to minimize $S$ at the two or three selected points in Figures 1 or 2 respectively. A more extensive comparison will appear elsewhere [16].

We acknowledge many discussions with S. Brouard. The work has been supported by Gobierno Autónomo de Canarias (Spain) (Grant No. PB2/95) and Ministerio de Educación y Cultura (Spain) (PB 93-0578). 


\section{REFERENCES}

[1] N. Balakrishnan, C. Kalyanaraman and N. Sathyamurthy, Phys. Rep. 280, 79 (1997).

[2] R. Kosloff R and D. Kosloff, J. Comp. Phys. 63, 363 (1986).

[3] D. Neuhauser and M. Baer, J. Chem. Phys. 92, 3419 (1990)

[4] D. Neuhauser, J. Chem. Phys. 103, 8513 (1995).

[5] T. Peng and J. Z. H. Zhang, J. Chem. Phys. 105, 6072 (1996); W. Zhu, T. Peng and J. Z. H. Zhang, J. Chem. Phys. 106, 1742 (1997).

[6] T. Seideman and W. H. Miller, J. Chem. Phys. 96, 4412 (1992).

[7] U. Peskin and W. H. Miller, J. Chem. Phys. 102, 4084 (1995).

[8] G. R. Allcock, Ann. Phys. 53, 253 (1969); Ann. Phys. 53, 286 (1969); Ann. Phys. 53, $311(1969)$.

[9] S. Brouard, D. Macías and J. G. Muga, J. Phys. A 27, L439 (1994).

[10] R. D. Levine and R. B. Bernstein, Molecular Reaction Dynamics and Chemical Reactivity, Oxford U. P., 1987.

[11] J. E. Beam, Am. J. Phys. 38, 1395 (1970).

[12] M. G. Rozman, P. Reineker and R. Tehver, Phys. Rev. A 49, 3310 (1994).

[13] For any isolated potential barrier the left and right transmission amplitudes are equal when the potential level is equal on both sides.

[14] For some numerical applications, a barrier with positive imaginary part may be problematic. In these cases it is necessary to minimize $f$ with the constraint $\operatorname{Im} V_{j}<0$

[15] D. Macías, S. Brouard, and J. G. Muga, Chem. Phys. Lett. 228, 672 (1994).

[16] J. P. Palao and J. G. Muga, to appear. 


\section{FIGURE CAPTIONS}

Figure 1. Survival $S(k)$ versus $k$. The thick solid and thick dotted lines correspond to "perfectly absorbing composite potentials" with $L_{2}=0.5$, and $L_{2}=1.6$ respectively $\left(L_{1}=1\right.$ in both cases), that absorb at $k_{1}=1$ and $k_{2}=1.2$. (For numerical stability the theoretical $V_{1}$ is truncated for values of the real or imaginary parts larger than $10^{3}$.) The dotted and dashed lines correspond to "square barrier composite potentials" with $N=2$ and $N=3$ respectively, with the same total length as the potential of the thick solid line, and optimized for the same values of $k(s=2)$. The solid line with circles corresponds to the potential $-i \eta x^{2}$, where $\eta$ minimizes the sum of the survivals at the two selected wavenumbers.

Figure 2. $S(k)$ versus $k$. The thick solid line corresponds to a "perfectly absorbing composite potential" constructed with three units to absorb at $k_{1}=1.94, k_{2}=4.84$, and $k_{3}=7.75, L_{1}=1, L_{2}=.008$, and $L_{3}=.024$. As in Figure 1 this potential is truncated to avoid numerical instability. Also shown is the survival for square barrier composite potentials optimized for the same points $(s=3)$, and with the same total length: $N=1$, solid line ; $N=2$, dotted line; $N=3$, dashed line; $N=4$, dotted-dashed line. The solid line with circles corresponds to the potential $-i \eta x^{2}$, where $\eta$ minimizes the sum of the survivals at the three selected wavenumbers. 


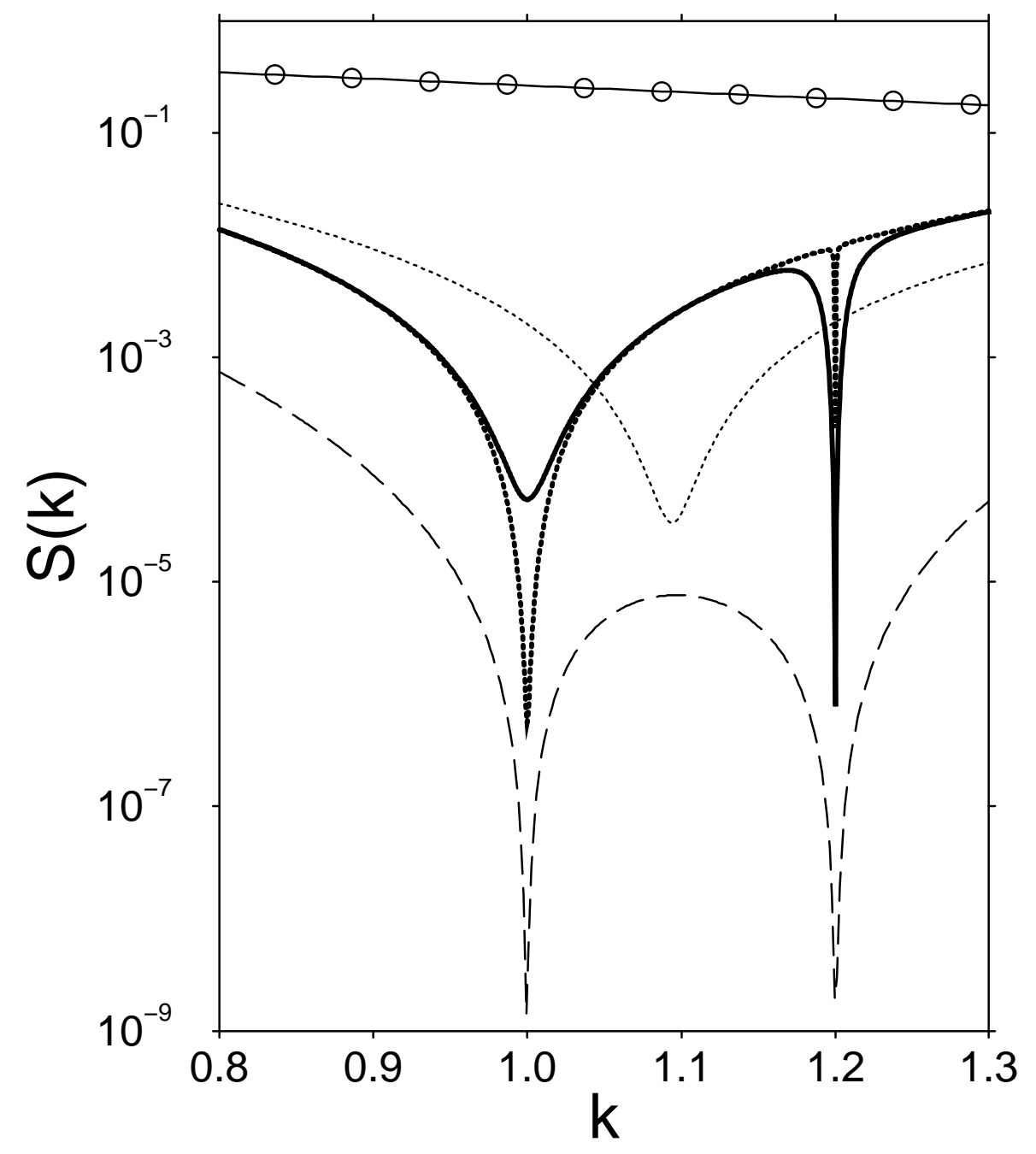




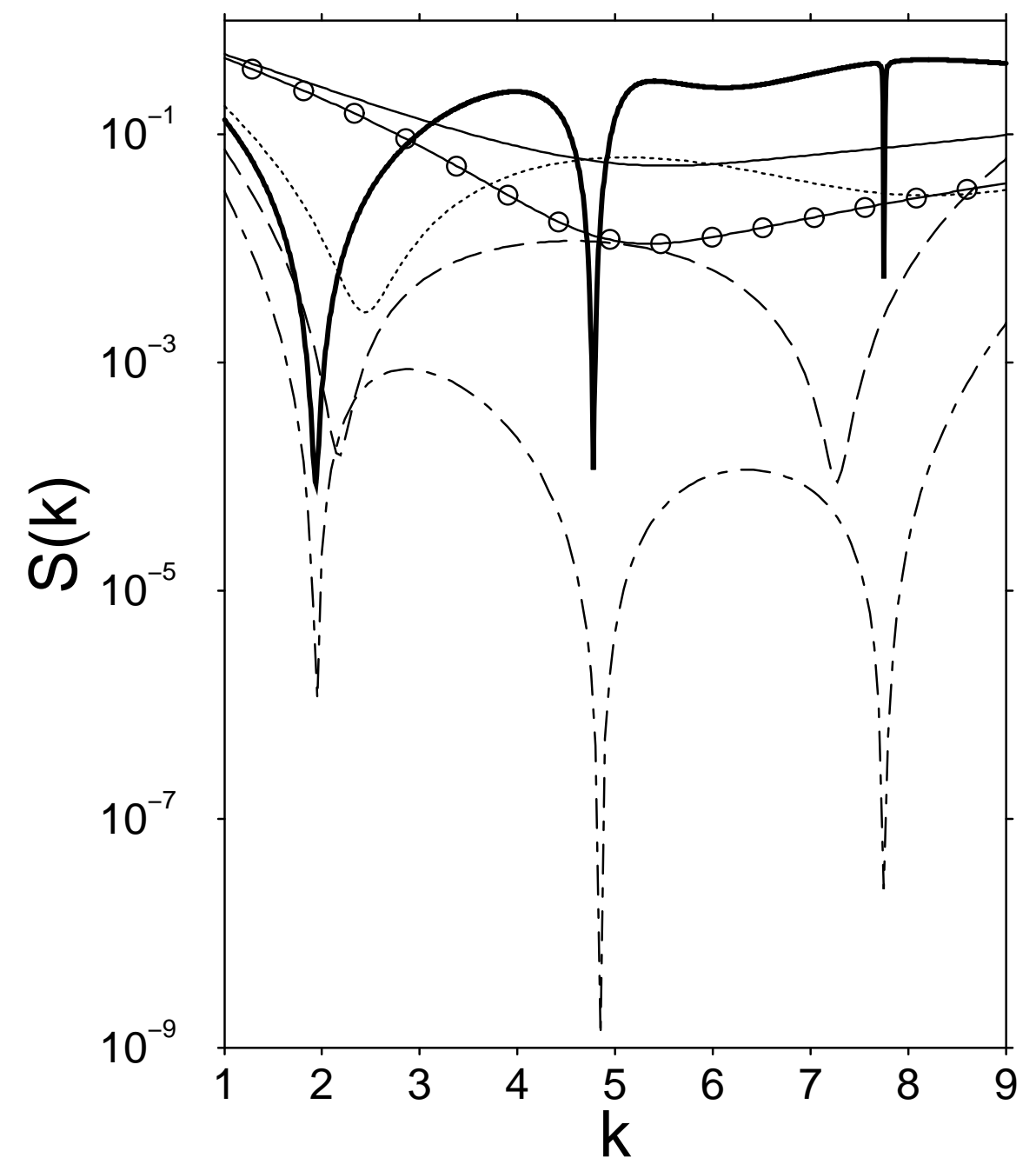

